\title{
Propiedades mecánicas de la dentina humana
}

\author{
Fuentes Fuentes $M^{a} V *$
}

\section{RESUMEN}

Puesto que la dentina es el mayor constituyente de la estructura dental, su microestructura y sus propiedades son los principales determinantes en casi todos los procedimientos de Odontología restauradora. Este artículo trata de las siguientes propiedades mecánicas de la dentina humana: la resistencia cohesiva, la microdureza y el módulo de elasticidad.

Palabras clave: Dentina, propiedades mecánicas, adhesión.

\section{ABSTRACT}

Since dentin is the major constituent of the teeth, dentin microstructure and their properties are principal determinants of nearly all procedures in restorative dentistry. This paper is about following mechanical properties of human dentin: cohesive strength, microhardness and modulus of elasticity.

Key words: Dentin, mechanical properties, adhesion.

Aceptado para publicación: octubre 2003.

* Becaria Predoctoral. Facultad de Odontología. Universidad de Granada.

Fuentes Fuentes Ma V. Propiedades mecánicas de la dentina humana. Av. Odontoestomatol 2004; 20-2: 79-83.

La dentina constituye la mayor parte de la estructura dental y sus propiedades son determinantes en casi todos los procedimientos de Odontología Restauradora (Marshall et al., 1997).

Es importante conocer las propiedades mecánicas de la dentina para entender cómo se distribuyen y absorben las fuerzas originadas durante la masticación y para predecir alteraciones que puedan sufrir debido a los procedimientos restauradores, la edad y la patología (Marshall et al., 1997).

Por otro lado, su conocimiento permite la confección de materiales restauradores que imiten sus propiedades (Xu et al., 1998). Igualmente, permitirá predecir el comportamiento de la interfase dentina/restauración (Marshall et al., 1997). Es en este último punto, en las implicaciones de las pro- piedades mecánicas en la adhesión a la dentina, en el que nos vamos a centrar.

Las propiedades de la dentina dependen básicamente de su estructura y composición.

Químicamente la dentina está compuesta alrededor de un $50 \%$ de su volumen de contenido mineral (cristales de hidroxiapatita ricos en carbonatos y pobres en calcio), de un $30 \%$ de su volumen de matriz orgánica, en su mayor parte colágena tipo I, y el $20 \%$ es fluido, similar al plasma sanguíneo, pero peor definido (LeGeros, 1990).

Su microestructura está dominada por la presencia de túbulos dentinarios. Los túbulos están rodeados por una región peritubular hipermineralizada, y que a su vez se haya embebida en una matriz intertubular 
formada principalmente por colágeno tipo I que engloba, configurando un entramado, cristales de hidroxiapatita y fluido dentinario. Los túbulos se extienden desde la cámara pulpar hasta la unión amelodentinaria. Estos canales varían en número y pueden representar desde el $1 \%$ (0.8 mm de diámetro) del área total de la superficie de la dentina junto a la unión amelodentinaria y aumentar en dirección a la pulpa hasta el 22\% (2.5 mm de diámetro) del área total de superficie de la dentina (Pashley et al., 1989). Esta organización determina un comportamiento anisotrópico de la dentina, es decir, las propiedades del substrato difieren según la dirección considerada.

Se han realizado numerosas estudios acerca de las propiedades mecánicas de la dentina. Desafortunadamente, los resultados obtenidos varían ampliamente. No está claro si los valores publicados reflejan diferencias en la estructura dental o son debidos a los métodos de ensayo empleados. En este artículo nos vamos a centrar en el estudio de la resistencia cohesiva, microdureza y módulo de elasticidad de la dentina.

\section{RESISTENCIA DE LA DENTINA}

La adhesión se puede definir como el estado en el que dos superficies se mantienen unidas mediante fuerzas o energías interfaciales basadas en mecanismos químicos, mecánicos o ambos, con la mediación de un adhesivo (ISO/TR 11405: 1994(E)). En Odontología son tres substratos los que participan en la adhesión: el material de restauración, el diente y el sistema adhesivo. Si sometemos a estrés la interfase dentina/restauración hasta el punto de fallo, la fractura puede localizarse en uno de los substratos anteriormente nombrados. Es decir, en la capa de adhesivo (fallo adhesivo), en la resina compuesta próxima a la interfase (fallo cohesivo), o bien en la dentina (fallo cohesivo). Normalmente el tipo de fallo es mixto. La localización en uno u otro substrato depende de las propiedades mecánicas de cada uno de los constituyentes, así como del espesor del agente adhesivo (Wakasa et al., 1995).

Algunos sistemas adhesivos actuales desarrollan grandes fuerzas de adhesión, superando la resistencia de la dentina y provocando que el fallo ocurra dentro del substrato. Por otro lado, durante los procedimientos de adhesión, la dentina es sometida a cambios en su composición. La mayoría de las técnicas actuales de adhesión utilizan un ácido para remover el barrillo dentinario, ensanchar la luz tubular y disolver los cristales de apatita que cubren la colágena de la dentina intertubular. Este tratamiento crea canales alrededor de las fibras que proporcionarán retención mecánica a los monómeros adhesivos hidrofílicos. El compuesto natural de apatita y colágeno se transforma en otro denominado Capa Híbrida, constituido por resina y colágeno. Las propiedades son diferentes de las originales y dependen de las propiedades de la colágena y de la resina (Sano et al., 1995).

La resistencia de la dentina tiene gran importancia para entender las características de los fallos adhesivos a la dentina (Watanabe et al., 1996).

Son numerosos los estudios realizados en este sentido. Bowen y Rodriguez (1962) publicaron que la resistencia de la dentina a la tensión se encuentra en torno a los $52 \mathrm{MPa}$, mientras que Lehman (1967) alrededor de los $37 \mathrm{MPa}$. Smith y Cooper (1971) publicaron valores de $39 \mathrm{MPa}$ en las proximidades de la cámara pulpar y de $131 \mathrm{MPa}$ cerca de la unión amelodentinaria utilizando una técnica de cizalla con punzón. Otros autores utilizaron el método de resistencia a la cizalla en un plano único (Gwinnet, 1994; Watanabe et al., 1996). Watanabe et al. (1996) determinó la resistencia de la dentina entre $78 \pm 13$ MPa y $91.8 \pm 12.7 \mathrm{MPa}$ dependiendo de la localización y de la orientación tubular. Estos autores observaron que los valores de resistencia de la dentina son mucho mayores que los valores de fuerza adhesiva utilizando el mismo método. Esto sugirió que los fallos localizados en la dentina en los estudios de adhesión podían ser el resultado de concentraciones de estrés debido a defectos en la superficie de adhesión Watanabe et al., (1996).

La técnica de microtensión desarrollada por Sano et al. (1994), permitió medir la resistencia de la dentina en superficies pequeñas $\left(0.5 \mathrm{~mm}^{2}\right)$. Los valores obtenidos con esta metodología fueron muy superiores a los valores que se habían obtenidos previamente por otros autores. Esto es debido a que al realizar mediciones en especímenes de pequeño tamaño, se con- 
sigue una mejor distribución de fuerzas, la aplicación de fuerzas de tracción puras y se evitan la presencia de defectos en la superficie que puedan alterar los resultados (Pashley et al., 1995).

Sano et al. determinaron la resistencia de la dentina mineralizada o intacta en $106 \mathrm{MPa}$ mientras que la resistencia del colágeno o dentina desmineralizada fue de tan solo de $29 \mathrm{MPa}$. La infiltración de la dentina desmineralizada con algunos sistemas adhesivos, restauró e incluso superó la resistencia de la dentina mineralizada (Sano et al., 1994 y 1995).

La resistencia de la dentina depende de la orientación tubular y es mayor cuando la carga se aplica perpendicular al eje axial de los túbulos (Carvalho et al., 2002). La explicación es desconocida pero probablemente se relaciona con la estructura de la dentina y la distribución de las fuerzas durante el ensayo. Kinney et al. (1996 y 1999) demostró que la dentina peritubular es más dura que la que la dentina intertubular. Si la fuerza que se aplica es paralela a la unión de estos dos componentes se producen fuerzas de cizalla. Por el contrario, si las fuerzas se aplican perpendicular a la orientación de los túbulos, las fuerzas generadas son de tensión. Se creen que las fuerzas de cizalla se propagan más rápidamente, provocando la fractura de la dentina con menor carga aplicada.

\section{DUREZA}

La dureza puede definirse como la resistencia de un material a la deformación permanente (Van Meerbeek et al., 1993) y se relaciona con otras propiedades como la generación de estrés y el módulo de Young. Existe una relación lineal entre la resistencia, módulo de elasticidad y la dureza (Perinka, Sano y Hosoda, 1992; Currey and Brear, 1990; Mahoney et al., 2000).

La mayoría de las mediciones de dureza en la dentina se realizan mediante las técnicas de microindentación Knoop y Vickers. Son técnicas rápidas de realizar y no destructivas. Los cálculos se basan en la deformación permanente inducida en la superficie que permanece tras la retirada de la carga (Van Meerbeek et al., 1993). En la prueba de durometría Vickers se utiliza un indentador piramidal de diaman- te con un ángulo diedro de $136^{\circ}$, mientras que en el procedimiento Knoop la punta del indentador es piramidal pero de base rómbica, formada por dos caras perpendiculares entre sí con ángulos de $172^{\circ}$ 30 ' y $130^{\circ}$ (Anusavice, 1996).

Los valores publicados de microdureza de la dentina oscilan entre los 250 y $800 \mathrm{MPa}$ dependiendo de la localización de la medida con respecto al esmalte y a la pulpa (Pashley et al., 1995; Craig et al., 1958). Pashley et al. (1985) observó una relación inversa entre la dureza de la dentina y la densidad tubular. Puesto que los ensayos de microindentación no permiten medir la dureza de estructuras individuales como la dentina peritubular e intertubular, el desarrollo de nuevas técnicas han permitido determinar si esta disminución de la dureza se debe a la morfología de la dentina o bien a diferencias en las propiedades de los constituyentes de la dentina.

El nanodurómetro emplea cargas entre $0.001-5 \mathrm{~g}$ (pocos mili-Newtons), resultando en indentaciones con profundidades inferiores a $1 \mathrm{~mm}$ en materiales duros. La profundidad de la indentación se monitoriza in situ durante la carga y la descarga, y se puede calcular la dureza y el módulo de Young a partir de la curva carga-desplazamiento sin necesitar información visual (Van Meerbeek et al., 1993). Sin embargo, esta técnica no permite la localización exacta en micras, haciendo difícil distinguir la dentina peritubular de la intertubular. Otro inconveniente es la imposibilidad de mantener los especímenes hidratados con lo que los valores obtenidos son mayores de los reales (Marshall et al., 1997).

La incorporación de un indentador en el microscopio de fuerzas atómicas ha permitido medir la dureza y rigidez de especímenes completamente hidratados con precisión de nanómetros (Kinney et al., 1996). De esta forma se ha medido la dureza de la dentina peritubular (de $2.45 \pm 0.14 \mathrm{GPa}$ ), que es varias veces más dura que la dentina intertubular. Su contenido en mineral es del $95 \%$ de su volumen, a diferencia del contenido de la dentina intertubular que es del $30 \%$ de su volumen. La dentina intertubular profunda es 4 veces más blanda que la dentina intertubular superficial $(0.51 \pm 0.02 \mathrm{GPa}$ cerca de la unión amelodentinaria y $0.13 \pm 0.01 \mathrm{GPa}$ cerca de pulpa) (Kinney et al., 1996). Por lo tanto se puede concluir 
que la disminución de la dureza de la dentina observada con las técnicas de microindentación, es debida a la disminución de dureza de la dentina intertubular y no a la mayor densidad de los túbulos de la dentina (Kinney et al., 1999).

\section{MÓDULO DE ELASTICIDAD}

El módulo de elasticidad o de Young puede definirse como el cociente entre la tensión aplicada a un material y la deformación elástica producida (es decir, que pueda recuperarse tras el cese de la carga).

La dentina mineralizada es relativamente rígida (10$20 \mathrm{GPa}$ ) (Sano et al., 1995). La elasticidad propia de la dentina tiene gran importancia funcional, ya que permite compensar la rigidez del esmalte, amortiguando los impactos masticatorios. La elasticidad dentinaria varía de acuerdo al porcentaje de sustancia orgánica y al agua que contiene. La microscopía de fuerzas atómicas ha demostrado que la dentina peritubular es mucho más rígida que la dentina intertubular y su módulo es más uniforme, mientras que el módulo de la dentina intertubular varía en función de la distribución de la apatita en la matriz de colágeno (Kinney et al., 1996).

Tras el grabado ácido, la matriz húmeda de dentina desmineralizada es más elástica $(5 \mathrm{MPa})$ (Maciel et al., 1996). Debido a la escasa rigidez, la red colágena puede colapsarse al secar con aire e interferir con la infiltración de monómeros. Los solventes orgánicos que componen los adhesivos tienen la función de reemplazar el contenido de agua de las fibras de colágena para facilitar la infiltración de la resina. A la vez deshidratan el colágeno, aumentando el módulo de elasticidad de la dentina expuesta y alterando la permeabilidad de la red colágena (Carvalho et al., 1996; Nakabayashi y Pashley, 1998).

Tras infiltrar la dentina desmineralizada con resina, el módulo de este nuevo compuesto supera el de la resina aunque sigue siento muy inferior al de la dentina intacta (Sano et al., 1995). Se ha publicado que el módulo de Young del colágeno en la interfase (entre la dentina y la interfase) es de 2 GPa y de 7 $\mathrm{GPa}$ en la región adyacente e incrementa hasta la normalidad conforme se aleja de la interfase (Katz et al., 2001). El bajo módulo elástico de la capa híbrida con respecto a la dentina, permite una capacidad de deformación suficiente para liberar el estrés de contracción de polimerización de la resina compuesta. Esto mejora la adhesión a la dentina, y por lo tanto la integridad marginal y la retención de la restauración (Van Meerbeek et al., 1993).

\section{BIBLIOGRAFÍA}

- Anusavice KJ. Mechanical properties of dental materials. In: Phillips' Science of Dental Materials. Mexico: McGraw-Hill Companies, Inc., 1996.

- Bowen RL, Rodriguez MS. Tensile strength and modulus of elasticity of tooth structure and several restorative materials. J Am Dent Assoc, 1962; 64: 378-87.

- Carvalho RM, Fernandes CAO, Villanueva R, Wang L, Pashley DH. Tensile strength of human dentin as function of tubule orientation and density. J Adhesive Dent, 2001; 3: 309-14.

- Craig RG, Peyton FA. The microhardness of enamel and dentine. J Dent Res, 1958; 37: 661-8.

- Carvalho RM, Yoshiyama M, Pashley EL, Pashley $\mathrm{DH}$. In vitro study on the dimensional changes of human dentine after demineralization. Arch Oral Biol 1996; 41: 369-77.

- Currey JD and Brear K. Young's modulus and yield strength in mammalian mineralized tissues. J Mater Sci Mater Med, 1990; 1: 14-20.

- Fusayama T, Okuse K, Hosoda H. Relationship between hardness, discoloration, and microbial invasion in carious dentin. J Dent Res, 1966; 15: 1033-46.

- Gwinnett AJ. A new method to test the cohesive strength of dentin. Quintessence Int, 1994; 25 : 215-8.

- International Organization for Standarization. 
ISO/TR 11405: 1994 (E) Dental materialsGuidance on testing adhesion to tooth structure. Ginebra, Suiza: ISO, 1994.

- Katz JL, Bumrerraj S, Dreyfuss J, Wang Y, Spencer P. Micromechanics of the dentin/adhesive interface. J Biomed Mater Res: Appl. Biomat, 2001; 58: 1366-71.

- Kinney JH, Balooch M, Marshall SJ, Marshall GW, Weih TM. Atomic Force Microscope measurements of the hardness and elasticity of peritubular and intertubular human dentin. J Biomechanical Engineering, 1996; 118: 133-5.

- Kinney JH, Balooch M, Marshall SJ, Marshall GW, Weih TM. Hardness and Young's modulus of peritubular and intertubular dentine. Arch Oral Biol 1996; 41: 9-13.

- Kinney JH, Balooch M, Marshall GW, Marshall SJ. A micromechanics model of the elastic properties of human dentine. Arch Oral Biol 1999; 44: 813-22.

- LeGeros. Chemical and crystallographic events in the caries process. J Dent Res 1990; 69: 567-74.

- Lehman ML. Tensile strength of human dentin. J Dent Res, 1967: 46: 197-201.

- Maciel KT, Carvalho RM, Ringle RD, Preston CCD, Russell CM, Pashley DH. The effects of acetone, ethanol, HEMA and air on the stiffness of human demineralized dentin. J Dent Res 1996; 75: 18518.

- Mahoney E, Holt A, Swain M, Kilpatrick N. The hardness and modulus of elasticity of primary molar teeth: an ultra-micro-indentation study. J Dent, 2000; 28: 589-94.

- Marshall GW, Marshall SJ, Kinney JH, Balooch M. The dentin substrate: structure and properties related to bonding. J Dent 1997; 25: 41-458.

- Nakabayashi N, Pashley DH. Hybridization of dental hard tissues. Ed. Quintessence, Tokio, 1998.

- Pashley D, Okabe A, Parham P. The relationship between dentin microhardness and tubule density. Endodontics and Dental Traumatology, 1985; 1: 176-9.

- Pashley DH. Dentin: a dynamic substrate. A review. Scan Microsc 1989; 3: 161-76.

- Pashley DH, Sano H, Ciucchi B, Yoshiyama M, Carvalho RM. Adhesion testing of dentin bonding agents: A review. Dent Mater 1995; 11: 117-25.

- Perinka L, Sano H, Hosoda H. Dentin thickness, hardness, and Ca-concentration vs bond strength of dentin adhesives. Dental Mater, 1992; 8: 22933.

- Sano H, Ciucchi B, Matthews WG, Pashley DH. Tensile properties of mineralized and demineralized human and bovine dentin. J Dent Res, 1994; 73: 1205-11.

- Sano H, Takatsu T, Ciucchi B, Russell CM, Pashley $\mathrm{DH}$. Tensile properties of resin-infiltrated demineralized human dentin. J Dent Res, 1995; 74: 1093102.

- Smith DC, Cooper WEG. The determination of shear strength. Brit Dent J, 1971; 130: 333-7.

- Van Meerbeek B, Willems G, Celis JP, Roos JR, Braem M, Lambrechts P, Vanherle G. Assessment by nano-indentation of the hardness and elasticity of the resin-dentin bonding area. J Dent Res 1993; 10:1434-42.

- Wakasa K, Yamaki M, Matsui A. Calculation models for average stress and plastic deformation zone size of bonding area in dentine bonding systems. Dent Mater J 1995; 14: 152-65.

- Watanabe L, Marshall GW, Marshall SJ. Dentin shear strength: effects of tubule orientation and intratooth location. Dent Mater 1996; 12: 109-15.

- Xu HHK, Smith DT, Jahanmir S, Romberg E, Kelly JR, Thompson VP, Rekow DE. Indentation Damage and Mechanical Properties of Human Enamel and Dentin. Journal of Dental Research, 1998; 77: 472-80. 